P. C. H. Martens, S. Tsuruta, and M. A. Weber, eds.

\title{
Particle Acceleration and Radiation in Magnetospheres of Collapsing Stars
}

\author{
V. Kryvdyk \\ Department of Astronomy, Kiev University, Glushkova 6, UKR-252022, \\ Kiev, Ukraine
}

\begin{abstract}
Particle dynamics and nonthermal emission therefrom in the magnetospheres of collapsing stars with initial dipole magnetic fields and a certain initial energy distribution of charged particles (power-law, relativistic Maxwell, and Boltzmann distributions) are considered . The radiation fluxes are calculated for various collapsing stars with initial dipole magnetic fields and an initial power-law particle energy distribution in the magnetosphere. The effects can be observed by means of modern instruments.
\end{abstract}

\section{Introduction}

Gravitational collapse starts when the mass of a stellar core exceeds the Chandrasekhar limit and the star becomes dynamically unstable. It is very difficult to observe the collapse. In this paper, a method of collapse observation using data of the electromagnetic radiation generated in the magnetosphere of the collapsing star is proposed.

\section{Magnetospheres of Collapsing Stars}

Particle dynamics and nonthermal emission from the magnetospheres of collapsing stars with initial dipole magnetic fields is considered. The dipole field is typical for stellar objects in their later evolution stages, such as white dwarfs and neutron stars (Shapiro \& Teukolsky 1985, Angel 1978). Charged particles in a magnetosphere have a certain initial energy distribution. In this paper, the three typical distributions in cosmic plasmas (power-law $N_{1}(E)=K_{C} E^{-\gamma}$, relativistic Maxwell $N_{2}(E)=K_{M} E^{2} \exp (-E / k T)$, and Boltzmann distributions $N_{3}(E)=K_{B} \exp (-E / k T)$ ) are considered. (Here, $K_{C}, K_{M}, K_{B}$ are the spectral coefficients, $k$ is the Boltzmann constant, and $T$ is the temperature.)

When a star's magnetosphere is compressed under collapse, its magnetic field increases considerably. The particle dynamics and the change of their spectrum in the magnetosphere are described by the equation for particle transitions in a regular magnetic field (Ginzburg \& Syrovatskii 1963):

$$
\frac{\partial N}{\partial t}+\frac{\partial}{\partial E}\left(N \frac{\mathrm{dE}}{\mathrm{dt}}\right)=0
$$


From this equation (Kryvdyk 1999),

$$
\begin{aligned}
& N_{1}^{\prime}(E, R)=K_{p} E^{-\gamma} R_{*}^{-\beta_{1}}, \\
& N_{2}^{\prime}(E, R)=K_{M} E^{2} R_{*}^{-\beta_{2}} e^{-\frac{E}{k T}}, \\
& N_{3}^{\prime}(E, R)=K_{B} R_{*}^{-\beta_{3}} e^{-\frac{E}{k T}}, \\
& N_{1}^{\prime \prime}(E, R)=K_{p} E^{-2 \gamma_{1}} e^{-\gamma\left(1-\gamma_{1}\right)}, \\
& N_{2}^{\prime \prime}(E, R)=K_{M} E^{2-4 \gamma_{1}} e^{-\left(1-\gamma_{1}\right) E / k T}, \quad \text { and } \\
& N_{3}^{\prime \prime}(E, R)=K_{B} E^{-2 \gamma_{1}} e^{-\left(1-\gamma_{1}\right) E / k T},
\end{aligned}
$$

where

$$
\begin{aligned}
\gamma_{1}= & \left(e^{4} F_{o}^{2} E / 6 m^{4} c^{7} r^{6}\right)\left(R R_{*} / 2 G M\right)^{1 / 2} F\left(R, R_{*}\right)\left(1+3 \cos ^{2} \theta\right) \sin ^{2} \alpha, \\
\beta_{1}= & (\gamma-1) f(\theta) \\
\beta_{2}= & (E / k T \ln E-3) f(\theta), \\
\beta_{3}= & (E / k T \ln E-1) f(\theta), \\
f(\theta)= & (5 / 3) k_{1}\left(3 \cos ^{4} \theta+1.2 \cos ^{2} \theta-1\right) /\left(1+3 \cos ^{2} \theta\right)^{2}, \quad \text { and } \\
F\left(R, R_{*}\right)= & R^{3}\left(R_{*}-1\right)^{1 / 2}\left(\frac{1}{3}+\frac{5}{12} R_{*}+\frac{5}{8} R_{*}^{2}\right)+ \\
& +\frac{5}{8} R^{3} R_{*}^{3} \arctan \left(R_{*}-1\right)^{1 / 2}
\end{aligned}
$$

The parameter $R_{*}=R_{o} / R$, where $R$ is the radius of a collapsing star with mass $M$ and initial radius $R_{o}$. The gravitational constant is $G ; \alpha$ is the angle between the magnetic field and the particle velocity, and $k_{1}=2,1$ for relativistic and nonrelativistic particles, respectively.

These equations determine the particle spectrum in the magnetosphere and its evolution during collapse for two cases: (1) when the energy losses do not influence the particle spectrum $\left(N_{1}^{\prime}, N_{2}^{\prime}, N_{3}^{\prime}\right)$, and (2) when energy loss is taken into account $\left(N_{1}^{\prime \prime}, N_{2}^{\prime \prime}, N_{3}^{\prime \prime}\right)$. The first case is typical for the initial stage of the collapse when the energy loss can be neglected. The second case is realized in the final stage of the collapse, when the magnetic field attains an extreme value and the energy losses considerably influence the particle spectrum.

\section{Nonthermal Emission from a Magnetosphere with a Power-law Particle Distribution.}

For the case when the distribution of particles is a power-law and the losses of energy do not influence the particle spectrum in the magnetosphere, the radiation flux has been determined (Kryvdyk 1999):

$$
I_{\nu}=I_{\nu o}\left(\nu / \nu_{0}\right)^{(1-\gamma) / 2} R_{*}^{\gamma-2}\left(I_{\nu 2} / I_{\nu 2 o}\right) .
$$


Here,

$$
\begin{aligned}
I_{\nu 2}\left(\theta, R_{*}, \gamma\right)= & \int\left(R_{*}\right)^{-\delta_{1}(\theta)} f\left(\theta, \theta_{0}, \varphi\right) \mathrm{d} \theta \mathrm{d} \varphi, \\
I_{\nu 2 o}\left(\theta, R_{*}=1, \gamma\right)= & \int f\left(\theta, \theta_{0}, \varphi\right) \mathrm{d} \theta \mathrm{d} \varphi, \\
\delta_{1}(\theta)= & \frac{\left(5 k_{1} / 3\right)(\gamma-2)\left(3 \cos ^{4} \theta+1.2 \cos ^{2} \theta-1\right)}{\left(1+3 \cos ^{2} \theta\right)^{2}}, \quad \text { and } \\
f\left(\theta, \theta_{o}, \varphi\right)= & \left\{\left(1+3 \cos ^{2} \theta\right)-\left[\left(3 \cos ^{2} \theta-1\right) \cos \theta_{0}+\right.\right. \\
& \left.\left.+3 \sin \theta \cos \theta \cos \varphi \sin \theta_{0}\right]^{2}\right\}^{(\gamma+1) / 4}(\sin \theta)^{4-3 \gamma},
\end{aligned}
$$

where $I_{\nu o}$ is the initial radiation flux from the collapsing stars for the fixed frequency $\nu_{o}$.

Using this equation, the radiation flux from the collapsing star can be calculated. The initial radiation flux from discrete radio sources is $10^{-22} \leq I_{\nu o} \leq$ $10^{-30} \mathrm{erg} / \mathrm{cm}^{2} \mathrm{~s} \mathrm{~Hz}$, the index $\gamma$ is in the range $2.2 \leq \gamma \leq 4$ (Ginzburg \& Syrovatskii 1963; Güdel \& Benz 1996; Utana, Trigilio, \& Catalano 1998). The numerical values of the radiation flux for the different $\gamma, R_{*}$, by $\left(\nu / \nu_{0}\right)=1$ and $\theta_{0}=0$, are given in Table 1 . For calculation, the maximum value $I_{\nu o}=$ $10^{-22} \mathrm{erg} / \mathrm{cm}^{2} \mathrm{~s} \mathrm{~Hz}$ was chosen. The other $I_{\nu i}$ can be calculated by the multiplication of the data from Table 1 with $I_{\nu i} / I_{\nu m}$.

\section{Conclusions}

The analysis of particle dynamics and its emission in a collapsing stellar magnetosphere show that the collapsing star can be a powerful source of nonthermal radiation which is produced by the interaction of charged particles with the magnetic field. The effect can be observed by means of modern instruments. The radiation flux grows with decreasing stellar radius and frequency, and will be observed in the form of pulsed radiation on a timescale like the typical duration of a stellar collapse. The flux depends on the distance to the star, the stellar magnetic field, and the particle spectrum in the magnetosphere. Thus, the observed radiation fluxes from different collapsing stars can differ from those given in Table 1.

Table 1. The value $\mathrm{I}_{\nu}$ for various $\mathrm{R}_{*}$ and $\gamma$ with $\left(\nu / \nu_{0}\right)=1$, $I_{\nu o}=10^{-22} \mathrm{erg} / \mathrm{cm}^{2} \mathrm{~s} \mathrm{~Hz}$, and $\theta_{0}=0$.

\begin{tabular}{llllll}
\hline$R_{*} / \gamma$ & 2.2 & 2.4 & 2.6 & 2.8 & 3.0 \\
\hline 100 & $3.17 \cdot 10^{-20}$ & $2.57 \cdot 10^{-19}$ & $2.14 \cdot 10^{-18}$ & $1.01 \cdot 10^{-27}$ & $1.54 \cdot 10^{-16}$ \\
200 & $1.17 \cdot 10^{-19}$ & $1.37 \cdot 10^{-18}$ & $1.65 \cdot 10^{-17}$ & $2.00 \cdot 10^{-16}$ & $2.47 \cdot 10^{-15}$ \\
400 & $4.47 \cdot 10^{-19}$ & $7.57 \cdot 10^{-18}$ & $1.31 \cdot 10^{-16}$ & $2.29 \cdot 10^{-15}$ & $4.07 \cdot 10^{-14}$ \\
600 & $9.90 \cdot 10^{-19}$ & $2.08 \cdot 10^{-17}$ & $4.45 \cdot 10^{-16}$ & $9.66 \cdot 10^{-15}$ & $2.12 \cdot 10^{-13}$ \\
800 & $1.75 \cdot 10^{-18}$ & $4.27 \cdot 10^{-17}$ & $1.06 \cdot 10^{-15}$ & $2.69 \cdot 10^{-14}$ & $6.87 \cdot 10^{-13}$ \\
1000 & $2.72 \cdot 10^{-18}$ & $7.48 \cdot 10^{-17}$ & $2.10 \cdot 10^{-15}$ & $5.96 \cdot 10^{-14}$ & $1.71 \cdot 10^{-12}$ \\
\hline \hline
\end{tabular}




\section{References}

Angel, J. R. P. 1978, ARAA, 16, 487

Ginzburg, V. L., \& Syrovatskii, S. I. 1963, Origin of cosmic rays (Moscow: Izdatel'stvo Akademiji Nauk USSR)

Güdel, M., \& Benz, A. O. 1996, in ASP Conf. Ser. Vol. 93, Radio Emission from the Stars and the Sun, eds. A. R. Taylor \& J. M. Paredes (Barcelona: ASP), 303

Kryvdyk, V. 1999, MNRAS, 309, 593

Shapiro, S. L., \& Teukolsky, S. A. 1985, Black Holes, White Dwarfs, and Neutron Stars (Moscow: Mir)

Utana, G., Trigilio, C., \& Catalano, S. 1998, AAP, 329, 1010 